ISSN 2519-7398 (Versión electrónica)

DOI: http://dx.doi.org/10.21704/ac.v78i1.862

(C) Universidad Nacional Agraria La Molina, Lma - Perú

\title{
Variables determinantes del perfil óptimo del ingresante al programa de doctorado en Economía de los Recursos Naturales-UNALM
}

\author{
Variables determining the optimum profile of students entering to the doctoral program at \\ economics of natural resources- UNALM
}

${ }^{1}$ Jorge Alarcón N., ${ }^{2}$ Rubén D. Miranda C.y ${ }^{3}$ Adriana Vílchez H.

\begin{abstract}
Resumen
El presente trabajo presenta los resultados de un estudio sobre el perfil optimo del ingresante al Programa de Doctorado en Economía de los Recursos Naturales y Desarrollo Sustentable (DERN-DS) de la UNALM. El propósito ha sido determinar la cantidad y calidad de las variables que determinan el éxito de los ingresantes al DERN-DS, para lo cual se hace uso de una propuesta metodológica relativamente novedosa, basada en un modelo Logit multinomial ordenado y un conjunto de pruebas no paramétricas. Los resultados obtenidos señalan a la formación académica previa en Ciencias Ambientales, la modalidad de trabajo y el ejercicio de la docencia, como variables relevantes que condicionan el perfil óptimo del estudiante, para su éxito en el Programa.
\end{abstract}

Palabras claves: perfil óptimo del ingresante; modelos probabilísticos; pruebas no paramétricas; posgrado en economía.

\begin{abstract}
This paper presents the results of a study on the optimal profile of incoming students to the Doctoral Program in Economics of Natural Resources and Sustainable Development (DERN-DS) of UNALM. The purpose has been to determine the quantity and quality of variables that determine the success of the entrants to the DERN-DS, for which a relatively new methodological proposal has been used, based on an ordered multinomial Logit model and a set of nonparametric tests. The obtained results point out the previous academic formation in environmental sciences, modality of work and the exercise of teaching, as relevant conditioning variables of the optimum profile of a new student.
\end{abstract}

Key words: Optimum profile of incoming students; Probabilistic models; Non-parametric tests; Postgraduate studies in economics.

\section{Introducción}

El sector educativo peruano viene atravesando una etapa crítica en la educación superior, rasgo que se manifiesta en niveles relativamente bajos de graduación de estudiantes de posgrado, situación que se presenta tanto en nuestro medio nacional como en otros contextos. Diferentes autores, como por ejemplo Chain (1995); Reyes (2006) y Sánchez (2006), citados por García y Barrón (2011), han hecho evidente la necesidad de saber más acerca de los estudiantes de posgrado, sus expectativas, sus intereses y motivaciones, así como sus problemas y necesidades. En la Universidad Nacional Agraria La Molina (UNALM), se presenta también baja graduación; del total de ingresantes a los programas de posgrado, menos del 10\% logran concluir sus estudios y obtener el grado esperado. Por otro lado, el perfil del estudiante a un determinado programa, ya sea de pregrado o posgrado, siempre es un elemento de singular importancia en la mejor decisión de los responsables de la admisión; el conocimiento de las características de dicho perfil no siempre es un elemento de sencilla determinación.

Esta investigación tuvo como objetivo general determinar las variables que contribuyen al perfil óptimo de los estudiantes del Programa de Doctorado en Economía de los Recursos Naturales y Desarrollo Sustentable (DERNDS) de la UNALM. Como objetivos específicos se han manejado: (i) determinar las variables determinantes, mediante el uso del modelo probabilístico Logit y pruebas no paramétricas, (ii) mostrar la aplicabilidad del análisis realizado, como una propuesta metodológica innovativa. Las variables que se utilizaron fueron de distinta naturaleza: académica, laboral y socio-demográfica.

Este artículo está compuesto de cinco secciones, incluida esta introducción. La segunda sección incluye una

\footnotetext{
'Departamento Académico de Economía y Planificación, Facultad de Economía y Planificación, Universidad Nacional Agraria La Molina, Lima, Perú. E-mail: jalarcon@lamolina.edu.pe

${ }^{2}$ Departamento Académico de Manejo Pesquero y Medio Ambiente, Facultad de Pesquería, Universidad Nacional Agraria La Molina, Lima, Perú. E-mail: mcruben@lamolina.edu.pe

${ }^{3}$ Departamento Académico de Economía y Planificación, Facultad de Economía y Planificación, Universidad Nacional Agraria La Molina, Lima, Perú.
} 
breve revisión de literatura, lo que permite entender mejor el contexto del trabajo. La tercera sección brinda información sobre los instrumentos metodológicos utilizados, mientras que la cuarta presenta los resultados, en línea con los objetivos. Finalmente, la quinta sección presenta una rápida discusión conclusiva del estudio.

No son muchos los estudios que, en la región, han enfatizado la modelística económica con el propósito de determinar el perfil óptimo de ingresantes a programas de estudios superiores. Entre los estudios revisados, destaca el trabajo de Bartual y Poblet (2009) quienes estudiaron los factores que inciden en resultados académicos de estudiantes de Ciencias Empresariales de la Universidad de Barcelona. Compararon dos grupos con diferentes modalidades de aprendizaje y utilizando un modelo de regresión logística, con una variable dependiente dicotómica, encontraron que, independientemente del tipo de enseñanza, la variable "asistencia a clases" siempre resultó significativa para entender el "éxito" del alumno. El estudio continuo, las sinergias asociadas al estudio en grupo y la preferencia en la elección de los estudios universitarios, fueron otros factores que mostraron una significativa incidencia en la probabilidad de aprobar el curso de Economía.

Asimismo, en la Universidad Católica del Norte (Chile), Soria y Zúñiga (2014) analizaron aspectos determinantes del éxito académico (egreso y titulación) de estudiantes de Ingeniería Comercial. Construyeron una base de datos de 564 estudiantes y 13 variables explicativas que, en un modelo Logit, permitió encontrar que las principales variables explicativas del éxito estudiantil, fueron las notas de la secundaria, el puntaje obtenido en el examen de admisión, así como el número de años de desfase de la educación media. A su turno, utilizando un modelo Logit semejante, Albalate, Fageda y Perdiguero (2011) analizaron el impacto en las calificaciones obtenidas por estudiantes de la Universidad de Barcelona, debido a la implantación de un cambio importante en metodologías docentes; concluyeron que una implementación exitosa de métodos educativos modernos mejoran substancialmente las calificaciones solo si se aumentan las horas que los alumnos dedican al estudio, también si se aumenta el porcentaje de asistencia y si se asegura que exista una coordinación adecuada de los diferentes programas de las asignaturas.

Martínez et al. (2009), también estudiaron el perfil óptimo del estudiante de posgrado de la Universidad Nacional Autónoma de México; utilizando la técnica estadística de Análisis Discriminante construyeron una ecuación de predicción múltiple que pronosticó un valor de probabilidad conocido si un alumno pertenecía al grupo de éxito académico (obtención del grado) o no formaba parte de él. Determinaron diferencias significativas entre los alumnos que obtuvieron el grado, y los que no lo hicieron, en seis variables de un total de 16 (dependientes económicos, empleo, número de semestres con beca durante el posgrado, monto de la beca, tipo de beca $\mathrm{y}$ tiempo de dedicación al posgrado). Luego, utilizando una probabilidad predictiva del 70\%, se estableció una variable determinante significativa para obtener el grado: el número de dependientes económicos.

\section{Materiales y métodos}

El estudio realizado es de tipo analítico, retrospectivo y con datos de corte transversal. La metodología sigue una secuencia de dos fases, la primera está basada en el uso de un modelo probabilístico (modelo Logit), que se caracteriza por tener la variable dependiente discreta multinomial, y se utilizó para determinar las probabilidades de ocurrencia de una situación de éxito (haber obtenido el grado académico), permitiendo esta fase clasificar los estudiantes de la muestra en 3 grupos: con mayor, menor y regular probabilidad de éxito. En la segunda fase se hace uso de un conjunto de métodos no paramétricos, entre los que destacan pruebas de homogenidad multivariada, de independencia bivariada (tablas de contingencia) y la prueba de KruskalWallis, usadas con el propósito de determinar el efecto diferenciado entre los grupos de clasificación.

\subsection{Modelo LOGIT ordinal ${ }^{4}$}

El modelo LOGIT es un modelo probabilístico que en general puede representase por:

$$
Y_{i}=X_{i} \beta+u_{i}
$$

Donde $\boldsymbol{X}_{i}$ es una matriz que representa las $k$-variables explicativas. $\boldsymbol{Y}_{\boldsymbol{i}}$ es la variable dependiente, discreta, que sigue una distribución de probabilidad (FDP) logística acumulativa, que permite expresar (1) a través de la siguiente expresión:

$$
F\left(Z_{i}\right)=F\left(X_{i} \beta\right)=\Lambda\left(X_{i} \beta\right)=P_{i}
$$

Para $P_{i}$, la función de probabilidad (FDP) puede representarse del siguiente modo:

$$
P_{i}=\frac{\exp \left(X_{i} \beta\right)}{1+\exp \left(X_{i} \beta\right)}=\frac{1}{1+\exp \left(-X_{i} \beta\right)}=1-\frac{\exp \left(-X_{i} \beta\right)}{1+\exp \left(-X_{i} \beta\right)}
$$

La forma operativa de la ecuación logística, que es estimada es:

$$
\operatorname{Li}=\operatorname{Ln}\left(P_{i} /\left(1-P_{i}\right)\right)=Z_{i} ; \text { donde } Z_{i}=\beta_{1}+\beta_{2} X_{2}+\ldots+\beta_{k} X_{k}
$$


La expresión (4) representa una ecuación semi-logarítmica (LOG-LIN) y los estimadores de los parámetros son semi-elasticidades, que expresan cambios relativos en la relación de probabilidades (de éxito respecto al fracaso), en función de cambios en valores absolutos (o sea considerando unidades de medida) de las variables regresoras involucradas.

El modelo LOGIT Ordinal se caracteriza por tener una variable dependiente multinomial, es decir con más de dos categorías y que es usado de manera ordinal (en forma ordenada); en el caso presente, por ejemplo, la variable dependiente tiene tres categorías ordenados jerárquicamente: (i) estudiantes que obtuvieron el grado doctoral, (ii) estudiantes que obtuvieron solo la candidatura, y (iii) los que no obtuvieron ninguna certificación.

Sea $\boldsymbol{Y}$ una variable de respuesta ordenada que toma valores $1,2,3, \ldots$, J. Entonces el modelo Logit ordenado para $\boldsymbol{Y}$ (condicionado a un conjunto de variables regresoras $\boldsymbol{X}$ ) se puede derivar de un modelo de variable latente:

$$
Y_{i}=X \beta+u_{i}
$$

Donde $\boldsymbol{X}$ no tiene término constante, $\beta$ contiene $k$ parámetros y $u_{i} / X \sim \mathrm{N}(0,1)$.

Siendo $\alpha_{1}<\alpha_{2}<\alpha_{3}$ puntos de corte desconocidos, entonces se define:

\begin{tabular}{ccc}
\hline$Y=0$ & $S i$ & $Y^{*} \leq \alpha_{1}$ \\
$Y=1$ & $S i$ & $\alpha_{1}<Y^{*} \leq \alpha_{2}$ \\
$Y=2$ & $S i$ & $\alpha_{2}<Y^{*} \leq \alpha_{3}$ \\
$\ldots$. & & $Y^{*} \leq \alpha_{j}$ \\
\hline
\end{tabular}

La distribución condicional de $\mathrm{Y}$ dado $\mathrm{X}$ viene dada por:

$$
\begin{aligned}
& P\left[Y_{i}=0 \mid X\right]=P\left[Y^{*} \leq \alpha_{1} \mid \mathrm{X}\right]=P\left(X \beta+u_{i} \leq \alpha_{1} \mid \mathrm{X}\right)=\Lambda\left(\alpha_{1}-X \beta\right) \\
& {\left[Y_{i}=1 \mid X\right]=P\left[\alpha_{1}<Y^{*} \leq \alpha_{2} \mid \mathrm{X}\right]=\Lambda\left(\alpha_{2}-X \beta\right)-\Lambda\left(\alpha_{1}-X \beta\right)}
\end{aligned}
$$

Finalmente: $P\left[Y_{i}=J \mid X\right]=P\left[Y^{*} \alpha_{j} \mid X\right]=1-\Lambda\left(\alpha_{j}-X \beta\right)$

El modelo Logit se estima usualmente por Máxima Verosimilitud. Los efectos marginales son los siguientes:

$$
\left(\partial P_{j}[X]\right) /\left(\partial X_{k}\right)=\beta_{\mathrm{k}}\left[v\left(\alpha_{(\mathrm{j}-1)}-X \beta\right)-\mathrm{v}\left(\alpha_{j}-X \beta\right)\right] ; 0<\mathrm{j}>\mathrm{J}
$$

\subsection{Pruebas no paramétricas}

Como pruebas no paramétricas se ha usado (i) la prueba de homogeneidad multivariada (o de atributos) y (ii) pruebas de independencia bivariada (tablas de contingencia) para determinar diferencias entre atributos de variables que pertenecen a estudiantes graduados y no graduados del DERN-UNALM. En ambos casos se usa un estadístico de prueba basado en la distribución Ji-cuadrada. Además (iii) la prueba no-paramétrica de Kruskal -Wallis. A continuación, se presentan las pruebas y las hipótesis, utilizando (como ejemplo) algunas variables del estudio. Se usa el término Ho para expresar la hipótesis nula e Ha en representación de la hipótesis alterna.

A. Prueba de Homogeneidad de atributos, multivariada.

\section{a. Planteamiento de hipótesis}

Ho: los graduados y los no graduados tienen un comportamiento homogéneo respecto a variados atributos. En la hipótesis alterna (Ha), los graduados y los no graduados tienen un comportamiento que difiere en al menos uno de los variados atributos.

\section{b. Estadístico de prueba}

Los grados de libertad se estiman como: $\&=(\mathrm{r}-1)(\mathrm{c}-1)$, con lo que se tiene:

$$
Q=\sum_{i=1}^{r} \sum_{j=1}^{c} \frac{\left(O_{i j}-E_{i j}\right)^{2}}{E_{i j}} \sim \chi_{(r-1)(c-1) G L}^{2}
$$

Donde: $\boldsymbol{Q}$ es el estadístico Ji-cuadrado

$\boldsymbol{O}_{i j}$ es la frecuencia observada

$\boldsymbol{E}_{i j}$ es la frecuencia esperada $\left.\left(n_{i} n_{j}\right) / N\right)$

$n_{i} n_{j}$ representa la suma total de la fila i por suma total de la columna $\mathrm{j}$

$\boldsymbol{N}$ es el tamaño total de la muestra.

c. Criterio de Decisión: con los grados de libertad \&=(r1). (c-1) y con un determinado nivel de significancia; se obtiene el valor Ji-cuadrado crítico (tabular) y se decide no rechazarla Ho si: Qcal $\leq$ Ji-cuadrado tabular, versus rechazar la Ho si: Qcal > Ji-cuadrado tabular.

\section{B. Prueba de Independencia bivariada (Tablas de Contingencia)}

Se realiza independientemente con cada una de las variables de interés en el estudio.

\section{a. Planteamiento de hipótesis}

Ho: La obtención del grado de doctor es independiente de la variable de interés. En la hipótesis alterna, la obtención del grado de doctor es dependiente de la variable de interés.

\section{b. Estadístico de prueba}

Los grados de libertad se estiman como: \&=(r-1) (c-1), con lo que se tiene:

$$
Q=\sum_{i=1}^{r} \sum_{j=1}^{c} \frac{\left(\left[O_{i j}-E_{i j}\right]-0.5\right)^{2}}{E_{i j}} \sim \chi_{(r-1)(c-1) G L}^{2}
$$


Donde: $\boldsymbol{Q}$ es el estadístico Ji-cuadrado

$\boldsymbol{O}_{i j}$ es la frecuencia observada

$\boldsymbol{E}_{i j}$ es la frecuencia esperada $\left.\left(n_{i} n_{j}\right) / N\right)$

$n_{i} n_{j}$ representa la suma total de la fila i por suma total de la columna $\mathrm{j}$

$\boldsymbol{N}$ es el tamaño total de la muestra.

c. Criterio de Decisión: con los grados de libertad \& $=(r-$ 1) (c-1) y con un determinado nivel de significancia, se obtiene el valor Ji-cuadrado crítico (tabular) y No se rechaza la Ho si: Qcal $\leq$ Ji-cuadrado tabular, versus rechazar Ho si: Qcal > Ji-cuadrado tabular.

\section{Prueba de Kruskal-Wallis (para variables cuantitativas) ${ }^{5}$}

Se aplica para comparar promedios de variables cuantitativas pertenecientes a dos o más muestras aleatorias tomadas en forma independiente, y con tamaños $n_{1}, n_{2}, \ldots, n_{k}$. Se parte de la premisa que todas las muestras provienen de una misma población y que, por tanto, los promedios de las subpoblaciones que se definen deben ser iguales. El adecuado funcionamiento de la prueba requiere (1) las sub-muestras de cada estrato sean aleatorias, (2) tamaños de sub-muestra dentro de cada estrato, mayor que cinco.

\section{a. Planteamiento de hipótesis}

Ho: Los promedios de las " $k$ " poblaciones son similares entre sí. En la hipótesis alterna (Ha) al menos uno de los promedios de las " $\mathrm{k}$ " poblaciones es diferente.

\section{b. Procedimiento}

Se ordenan las observaciones de menor a mayor, luego se les asigna un número de orden o rango. En caso de empates (observaciones que tienen el mismo valor), el rango que se asigne a dichas observaciones será el promedio de los rangos de las observaciones empatadas). Ti es la suma de los rangos del estrato o grupo "i”.

\section{c. Estadístico de prueba}

Los grados de libertad se estiman como: \&=(k-1), con lo que se tiene:

$$
Q=\frac{12}{n(n+1)} \sum_{i=1}^{k} \frac{T_{i}^{2}}{n_{i}}-3(n+1) \sim \chi_{(k-1) G L}^{2}
$$

Donde: $\boldsymbol{Q}$ es el estadístico Ji-cuadrado

$n_{i}$ tamaño de la sub-muestra de la población "i"

$\boldsymbol{T}_{\boldsymbol{i}}$ es la suma de rangos de la muestra en la población "i".

$n$ número total de observaciones $\left(n_{1}+n_{2} \ldots+n_{k}\right)$

$\boldsymbol{N}$ es el tamaño total de la muestra. c. Criterio de Decisión: con los grados de libertad $\&=(\mathrm{k}-$ 1) y con un determinado nivel de significancia, se obtiene el valor Ji-cuadrado crítico (tabular) y No se rechaza la Ho si: Qcal $\leq$ Ji-cuadrado tabular, versus rechazar la Ho si: Qcal > Ji-cuadrado tabular.

\section{Resultados y discusión}

\subsection{De los Modelos Probabilísticos}

Los resultados de los modelos de regresión probabilísticos son mostrados en la Tabla 1. Nótese que se obtuvieron 5 variables significativas $(\alpha=0.10)$ : estudios en economía, estudios en ciencias ambientales, trabajo independiente, docencia a tiempo completo y promedio ponderado acumulado $(\mathrm{n}=38)$.

Tabla 1. Justificación de variables independientes y añadido de la Var. Dependiente

\begin{tabular}{|c|c|c|}
\hline \multicolumn{2}{|c|}{ Variable Dependiente: categórica ordinal* } & \multirow{3}{*}{$\begin{array}{c}\text { Efecto } \\
\text { marginal } \\
\text { (P-valor) }\end{array}$} \\
\hline Variables Inde & pendientes & \\
\hline \multicolumn{2}{|l|}{ Variables } & \\
\hline ECON & Estudios Economía & $\begin{array}{c}3.150678 \\
(0.0031) \\
\end{array}$ \\
\hline EAMB & Estudios Ciencias Ambientales & $\begin{array}{r}1.486307 \\
(0.0593) \\
\end{array}$ \\
\hline TRABINDEP & Trabajo Independiente & $\begin{array}{c}-1.402425 \\
(0.0562)\end{array}$ \\
\hline DOCCOM & Docencia a tiempo Completo & $\begin{array}{r}-2.12719 \\
(0.0297) \\
\end{array}$ \\
\hline PROM & Promedio Ponderado Acumulado & $\begin{array}{c}0.847935 \\
(0.0874)\end{array}$ \\
\hline COMI & Comité Mixto o NO & $\begin{array}{c}1.921 \\
(0.2191) \\
\end{array}$ \\
\hline $\mathbf{S X}$ & Sexo & $\begin{array}{c}0.92719 \\
(0.297) \\
\end{array}$ \\
\hline NAC & Nacionalidad & $\begin{array}{c}1.2119 \\
(0.1597) \\
\end{array}$ \\
\hline CRED & Créditos Aprobados Acumulados & $\begin{array}{l}0.02167 \\
(0.3421)\end{array}$ \\
\hline $\mathbf{R}$ - cuadrado & & 0.31688 \\
\hline $\mathrm{X}^{2}$ cuadrado & & 2.75176 \\
\hline (p-value) & & 0.08576 \\
\hline $\mathrm{X}^{2}$ cuadrado & & 2.75176 \\
\hline (p-value) & & 0.08576 \\
\hline
\end{tabular}

Luego de haber obtenido los resultados del modelo de regresión Logit multinomial, se pronosticó las probabilidades de obtención del Doctorado y las probabilidades de no obtención del grado, por cada uno de los individuos (es decir en cada uno de los casos o de las observaciones). Luego se ordenaron las observaciones de acuerdo a sus probabilidades, de mayor a menor (de haber obtenido el grado de doctor, de haber obtenido solo 
la candidatura y la tercera opción de no haber obtenido ninguno de los dos diplomas).

Después del ordenamiento de las probabilidades, se tomó el grupo de estudiantes ubicados en el tercio superior (estudiantes con la más alta probabilidad de obtención del grado académico doctoral), en contraposición con los estudiantes del tercio inferior (estudiantes con probabilidad de no obtención de ningún grado doctoral). En base a estos dos grupos de estudiantes, se llevaron a cabo el conjunto de pruebas no paramétricas que han permitido establecer diferencias (estadísticamente significativas) entre un grupo y otro, con el propósito de definir fundamentalmente el perfil de estudiantes con la más alta probabilidad de culminar sus estudios exitosamente.

\subsection{Pruebas no paramétricas}

\section{A. Pruebas de homogeneidad multivariada}

Las pruebas no paramétricas de homogeneidad comprendieron evaluaciones de hipótesis que son multivariadas; en el presente caso éstas se hicieron en dos grupos de variables: estudios previos (en economía y ciencias ambientales) y tipo de trabajo, en el primer grupo. En el segundo grupo se evaluaron, en forma conjunta las variables: sexo, docente a tiempo parcial, docente a tiempo completo, estudiantes con comité mixto y nacionalidad.

Tabla 2. Resultados de Pruebas de Homogeneidad Multivariada

\begin{tabular}{|c|c|c|c|}
\hline & \multicolumn{2}{|c|}{$\begin{array}{c}\text { Estudiantes que } \\
\text { obtuvieron }\end{array}$} & \multirow[b]{2}{*}{$\chi^{2}$ calculada } \\
\hline & $\begin{array}{l}\text { Grado } \\
\text { Doctoral }\end{array}$ & $\begin{array}{l}\text { Ningún } \\
\text { grado }\end{array}$ & \\
\hline Estudios en Economía & $\begin{array}{c}9 \\
69 \%\end{array}$ & $\begin{array}{c}9 \\
69 \%\end{array}$ & \\
\hline $\begin{array}{l}\text { Estudios en Ciencias } \\
\text { Ambientales }\end{array}$ & $\begin{array}{c}8 \\
62 \% \\
\end{array}$ & $\begin{array}{c}2 \\
15 \% \\
\end{array}$ & \\
\hline Trabajo Independiente & $\begin{array}{c}5 \\
38 \% \\
\end{array}$ & $\begin{array}{c}10 \\
77 \%\end{array}$ & $5.25^{*}$ \\
\hline Sexo (No. De varones) & $\begin{array}{c}8 \\
62 \% \\
\end{array}$ & $\begin{array}{c}8 \\
62 \% \\
\end{array}$ & \\
\hline $\begin{array}{l}\text { Docente de Tiempo } \\
\text { Parcial }\end{array}$ & $\begin{array}{c}7 \\
54 \%\end{array}$ & $\begin{array}{c}0 \\
0 \%\end{array}$ & \\
\hline $\begin{array}{l}\text { Docente de Tiempo } \\
\text { Completo }\end{array}$ & $\begin{array}{c}5 \\
38 \% \\
\end{array}$ & $\begin{array}{c}11 \\
85 \%\end{array}$ & \\
\hline $\begin{array}{l}\text { Estudiantes con Comité } \\
\text { Tutor Mixto }\end{array}$ & $\begin{array}{c}8 \\
62 \%\end{array}$ & $\begin{array}{c}7 \\
54 \%\end{array}$ & \\
\hline $\begin{array}{l}\text { Nacionalidad (No. De } \\
\text { estudiantes Peruanos) }\end{array}$ & $\begin{array}{c}6 \\
46 \% \\
\end{array}$ & $\begin{array}{c}5 \\
38 \% \\
\end{array}$ & $9.29 *$ \\
\hline Tamaños de muestra & 13 & 13 & \\
\hline
\end{tabular}

* Estadístico significativo con $90 \%$ de confianza. $(\alpha=0,10)$.

Respecto a la evaluación del primer grupo de variables, las hipótesis fueron las siguientes:

Ho: Los graduados y no graduados tienen comportamiento homogéneo respecto a los atributos: estudios en economía, estudios en ciencias ambientales y trabajo independiente. En la Ha: los graduados y no graduados difieren en al menos uno de los atributos.

Considerando un nivel de confianza de 0,9 y con los datos observados en la Tabla 2, se calcularon las frecuencias esperadas y observadas (2 grados de libertad), obteniéndose un valor tabular Ji-cuadrada de 4,61. Como Qcal es 5,246, se rechazó la Ho. Y se concluyó que no hay un comportamiento estadísticamente homogéneo entre el grupo de estudiantes que se graduaron de Doctor y el grupo de aquellos que no obtuvieron ningún grado Doctoral; es decir difieren en al menos uno de los atributos de las variables.

Con respecto a la evaluación del segundo grupo de variables, las hipótesis fueron:

Ho: Los graduados y los no graduados tienen un comportamiento homogéneo respecto a los atributos: sexo, docente de tiempo parcial, docente de tiempo completo, estudiantes con comité tutor mixto y nacionalidad. En la hipótesis alterna (Ha): los graduados y los no graduados tienen un comportamiento que difiere en al menos uno de los atributos: sexo, docente parcial, docente completo, tipo de comité asesor de tesis y nacionalidad peruana.

Siendo el nivel de significancia igual a 0,10 (4 grados de libertad), se obtuvo un valor Ji-cuadrado tabular de 7,78 y un valor calculado (Qcal) de 9,29, entonces se rechazó la Ho y se concluye que los graduados (graduación completa), respecto a los no graduados, difieren en al menos una de las características evaluadas antes indicadas (Tabla 2).

En resumen, los resultados de hipótesis de evaluación conjunta de variables que influirían en los diferenciales entre estudiantes que obtuvieron el grado doctoral y aquellos que no lo hicieron, indican que efectivamente existen diferencias significativas entre ambos grupos de estudiantes en al menos una variable diferencial.

\section{B. Pruebas de independencia bivariada (Tablas de Contingencia).}

Estas pruebas de independencia son más específicas que las que se han realizado en la sección anterior, permitieron determinar la significancia estadística en forma más precisa y solo considerando variables individuales, las mismas que aparecen resumidas en la Tabla 3.

A continuación, en cada caso, se presentan la hipótesis nula o planteada (la hipótesis alterna es la opuesta) y los resultados de la prueba.

\section{Variable 1: Estudios de Economía}

Ho: La obtención del grado de doctor es independiente de que el estudiante tenga una base en estudios de ciencias económicas. En la hipótesis alterna $(\mathrm{Ha})$ no hay tal independencia.

En la Tabla 3 se muestran las frecuencias y porcentajes de ambos grupos. Con un nivel de confianza igual a 0,9 
y los datos observados en la referida Tabla, el valor Jicuadrado tabular es 2,71 y el valor calculado Qcal $=0,18$, con lo cual se concluyó que la obtención del grado de doctor es independiente de que el estudiante tenga una base en estudios de Economía. Es decir, que los estudios en Economía no influyen en la obtención del grado.

\section{Variable 2: Estudios en Ciencias Ambientales}

Ho: La obtención del grado de doctor es independiente de que el estudiante tenga una base en estudios de ciencias ambientales. En la hipótesis alterna no hay tal independencia.

La Tabla 3 muestra las frecuencias y porcentajes de ambos grupos. Con un nivel de confianza de 0,9 , y los datos observados en la Tabla, se calcularon las frecuencias esperadas. Dados los grados de libertad y como el valor calculado de Ji-cuadrado es Qcal $=4,06$, se rechazó la Ho y se concluye que la obtención del grado de doctor depende de que el estudiante tenga estudios previos en ciencias ambientales.

\section{Variable 3: Tipología de trabajo}

Ho: La obtención del grado de doctor es independiente de que el estudiante tenga un trabajo independiente. En la hipótesis alterna no hay tal independencia.

En la Tabla 3 se muestran las frecuencias y porcentajes de ambos grupos. Con el nivel de confianza de 0,9 y los datos observados y esperados, así como los grados de libertad (1), Ji-cuadrado tabular fue 2,71 y el calculado (Qcal) es 2,52, entonces no se recházó la Ho. Es decir, la obtención del grado de doctor no depende del hecho que el estudiante tenga un trabajo independiente.

Tabla 3. Resultados de Pruebas de Independencia Bivariada

\begin{tabular}{|c|c|c|c|}
\hline \multirow{2}{*}{ Variables dicotómicas } & \multicolumn{2}{|c|}{$\begin{array}{c}\text { Estudiantes que } \\
\text { obtuvieron }\end{array}$} & \multirow[b]{2}{*}{$\begin{array}{c}\chi^{2} \\
\text { calculada }\end{array}$} \\
\hline & $\begin{array}{c}\text { Grado } \\
\text { Doctoral }\end{array}$ & $\begin{array}{l}\text { Ningún } \\
\text { grado }\end{array}$ & \\
\hline \multirow[b]{2}{*}{ Estudios previos de Economía } & 9 & 9 & \\
\hline & $69 \%$ & $69 \%$ & \\
\hline \multirow{2}{*}{$\begin{array}{l}\text { No estudió Economía } \\
\text { (previamente) }\end{array}$} & 4 & 4 & \\
\hline & $31 \%$ & $31 \%$ & $0.18^{\mathrm{ns}}$ \\
\hline \multirow{2}{*}{$\begin{array}{l}\text { Estudios previos en Ciencias } \\
\text { Ambientales }\end{array}$} & 8 & 2 & \\
\hline & $62 \%$ & $15 \%$ & \\
\hline \multirow{2}{*}{$\begin{array}{l}\text { No Estudios de Ciencias } \\
\text { Ambientales }\end{array}$} & 5 & 11 & \\
\hline & $38 \%$ & $85 \%$ & $4.06^{*}$ \\
\hline \multirow{2}{*}{ Tiene Trabajo Independiente } & 5 & 10 & \\
\hline & $38 \%$ & $77 \%$ & \\
\hline \multirow{2}{*}{ No tiene Trabajo Independiente } & 8 & 3 & \\
\hline & $62 \%$ & $13 \%$ & $2.50^{\mathrm{ns}}$ \\
\hline \multirow{2}{*}{ Docente de Tiempo Parcial } & 7 & 0 & \\
\hline & $54 \%$ & $0 \%$ & \\
\hline \multirow{2}{*}{ No Docente de Tiempo Parcial } & 6 & 13 & \\
\hline & $46 \%$ & $100 \%$ & $0.21^{\mathrm{ns}}$ \\
\hline \multirow{2}{*}{ Sexo (Masculino) } & 8 & 8 & \\
\hline & $62 \%$ & $62 \%$ & \\
\hline Sexo (Femenino) & $\frac{5}{38 \%}$ & $38 \%$ & $0.002^{\text {ns }}$ \\
\hline
\end{tabular}

Continuación de tabla

\begin{tabular}{|c|c|c|c|}
\hline \multirow{2}{*}{ Docente de Tiempo Completo } & 5 & 11 & \multirow[b]{4}{*}{$7.04 *$} \\
\hline & $38 \%$ & $85 \%$ & \\
\hline \multirow{2}{*}{$\begin{array}{l}\text { No Docente de Tiempo } \\
\text { Completo }\end{array}$} & 8 & 2 & \\
\hline & $62 \%$ & $15 \%$ & \\
\hline \multirow{2}{*}{$\begin{array}{l}\text { Estudiante con Comité asesor } \\
\text { de tesis Mixto }\end{array}$} & 8 & 7 & \multirow[b]{4}{*}{$4.06^{*}$} \\
\hline & $62 \%$ & $54 \%$ & \\
\hline \multirow{2}{*}{$\begin{array}{l}\text { Estudiante con Comité asesor } \\
\text { de tesis No Mixto }\end{array}$} & 5 & 6 & \\
\hline & $38 \%$ & $46 \%$ & \\
\hline \multirow{2}{*}{ Nacionalidad Peruana } & 6 & 5 & \multirow{5}{*}{$0.010^{\mathrm{ns}}$} \\
\hline & $46 \%$ & $38 \%$ & \\
\hline \multirow{2}{*}{ Nacionalidad Extranjera } & 7 & 8 & \\
\hline & $54 \%$ & $62 \%$ & \\
\hline Tamaño de muestra & 13 & 13 & \\
\hline
\end{tabular}

\section{Variable 4: Sexo}

Ho: La obtención del grado de doctor es independiente de que el estudiante sea de sexo masculino o femenino. En la hipótesis alterna no hay tal independencia.

En el caso de esta variable, los resultados parecen ser evidentes, pues no hay mayor diferencia entre hombres y mujeres en ambos grupos (Tabla 3). Con el nivel de confianza de 0,9 y los datos observados y esperados, así como los grados de libertad (1), Ji-cuadrado tabular fue 0,002 y el calculado (Qcal) es 2,52, entonces no se rechazó la Ho. Es decir, la obtención del grado de doctor no depende del sexo. En tal sentido solo es posible concluir que la variable "Sexo" no afecta en nada el hecho que los doctorantes obtengan el grado académico de Doctor (o no).

\section{Variable 5: Docente a Tiempo Parcial}

Ho: La obtención del grado de doctor es independiente de que el estudiante sea docente -o no- a tiempo parcial o que no lo sea. En la hipótesis alterna no hay tal independencia.

La Tabla 3 muestra las frecuencias y porcentajes de ambos grupos. Con un nivel de significancia igual a 0,90 , el número de grados de liberta será de 1 y el valor Jicuadrado calculado es 7,04 (valor tabular 2,71); por tanto, se rechazó la Ho y se concluyó que la obtención del grado de doctor si depende de que el estudiante sea docente de tiempo parcial.

\section{Variable 6: Docente a Tiempo Completo}

Ho: La obtención del grado de doctor es independiente de que el estudiante sea docente -o no- a tiempo completo o que no lo sea. En la hipótesis alterna no hay tal independencia.

La Tabla 3 muestra las frecuencias y porcentajes de ambos grupos. Con los grados de libertad en 1, como Qcal es 4,06 (valor tabular 2,71), el criterio de decisión resultó en rechazar la Ho; se concluyó, por tanto, que la obtención del grado de doctor es dependiente de que el estudiante sea docente a tiempo completo o que no lo sea.

\section{Variable 7: Tipología del Comité asesor}

Ho: La obtención del grado de doctor es independiente de que el estudiante tenga un comité asesor (de tesis) mixto 
o no. En la hipótesis alterna no hay tal independencia.

La Tabla 3 muestra las frecuencias y porcentajes de ambos grupos. Con un nivel de significancia de 0,10 y con 1 grado de libertad, se obtiene un valor Ji-cuadrado tabular de 2,71 y un valor calculado (Qcal) de 0,010; entonces no se rechazó la Ho. La obtención del grado de doctor es independiente de que el estudiante tenga un comité asesor mixto o no.

\section{Variable 8: Nacionalidad}

Ho: La obtención del grado de doctor es independiente de que el estudiante tenga nacionalidad peruana o extranjera. En la hipótesis alterna no hay tal independencia.

La Tabla 3 muestra las frecuencias y porcentajes de ambos grupos. Dado el nivel de significancia igual a 0,10 y los grados de libertad es $1(n=26)$, entonces como el valor Ji-cuadrado tabular es 2,71 y el valor calculado (Qcal) es 0,001 , por lo que no se rechazó la Ho, concluyéndose que la obtención del grado de doctor es independiente de que el estudiante tenga nacionalidad peruana o extranjera. La nacionalidad no hace diferencia en cuanto a la culminación de estudios ni obtención del grado doctoral.

\section{Prueba Kruskal-Wallis}

Todos los resultados relacionados con este tipo de prueba estadística no paramétrica son mostrados en la Tabla 4.

Tabla 4. Resultados de pruebas Kruskal-Wallis

\begin{tabular}{cccccc}
\hline \multicolumn{6}{l}{ Variable: Promedios ponderados } \\
\hline Doctor & Rango & Candidato & Rango & No Grado & Rango \\
$\mathrm{T}$ & 279.0 & $\mathrm{~T}$ & 267.5 & $\mathrm{~T}$ & 233.5 \\
$\mathrm{~N}$ & 13 & $\mathrm{~N}$ & 13 & $\mathrm{~N}$ & 13 \\
Promedio & 17.13 & Promedio & 17.10 & Promedio & 16.83 \\
\hline Ji-cuadrada: $0,660^{n s}$ & \multicolumn{5}{c}{} \\
\hline \multicolumn{5}{l}{ Variable: Total de créditos académicos acumulados } \\
\hline Doctor & Rango & Candidato & Rango & No Grado & Rango \\
$\mathrm{T}$ & 274 & $\mathrm{~T}$ & 278.5 & $\mathrm{~T}$ & 223 \\
$\mathrm{~N}$ & 13 & $\mathrm{~N}$ & 13 & $\mathrm{~N}$ & 13 \\
Promedio & 52.77 & Promedio & 52.77 & Promedio & 47.77 \\
\hline
\end{tabular}

Ji-cuadrada: $0,001^{\text {ns }}$

* Estadístico significativo con $90 \%$ de confianza. $(\alpha=0,10)$

$n s$ Estadístico no significativo con $90 \%$ de confianza $(\alpha=0,10)$

$\mathrm{Ti}$ es la suma de los rangos del estrato o grupo “i”. $\mathrm{N}$ tamaño total de muestra.

\section{Caso 1. Promedio ponderado acumulado}

Ho: los promedios ponderados son similares entre las categorías: estudiantes con doctorado, estudiantes candidatos y aquellos estudiantes que no obtuvieron ningún grado. Ha: Los promedios difieren al menos entre una de las categorías.

Con un nivel de significancia igual a 0,10 y los datos observados para esta variable, en la Tabla 4 se presenta el cálculo de los rangos y sumas de rango de cada bloque. Con 2 grados de libertad, dado que Qcal es 0,66 y el valor Ji-cuadrado tabular es 4,61, se rechazó la Ho. Las evidencias muéstrales indican que los promedios académicos de notas de los alumnos en las 3 categorías (grado de doctor, obtención de candidatura y ningún grado) son similares.

\section{Caso 2. Número total de Créditos}

Ho: El número de créditos aprobados son similares entre las categorías: estudiantes con doctorado, candidatos y aquellos que no tienen ningún grado. En la hipótesis alterna el número de créditos aprobados difieren entre, al menos, una de las categorías.

Con el nivel de significancia igual a 0,10 , se calcularon los rangos y sumas de rango de cada bloque, tal como es mostrado en la Tabla 4. Con 2 grados de libertad, se obtuvo un valor de Q tabular de 4,61 y Qcal es 0,001, por lo que no se rechazó la Ho. Las evidencias muéstrales indican que el número de créditos aprobados son similares entre las categorías: con grado de doctor, candidatura y ningún grado.

\section{Conclusiones}

a. En general, desde el punto de vista metodológico, el modelo LOGIT, de naturaleza probabilística, permitió discriminar (en una primera fase) las variables relevantes, del total de variables consideradas. De tal modo fue posible discriminar la muestra en categorías de estudiantes, en función a la probabilidad (alta, intermedia o baja) de obtener el grado doctoral.

b. Producto del uso del Modelo Logit, para este estudio, fueron significativas las siguientes variables: estudios en economía, estudios en ciencias ambientales, trabajo independiente, docencia a tiempo completo y promedio ponderado acumulado. Predicciones derivadas de este análisis permitió determinar tres grupos de estudiantes, de acuerdo a los que tuvieron mayor probabilidad de haber obtenido el grado doctoral, los que tuvieron una menor probabilidad del grado doctoral y los que tienen una probabilidad intermedia.

c. Las pruebas no paramétricas han funcionado como un instrumento metodológico puntual, de detalle, en el establecimiento de diferencias (significativas o no, desde el punto de visto estadístico) entre grupos de estudiantes en las dos diferentes categorías establecidas previamente en base a la probabilidad de graduación.

d. Con uso de pruebas no paramétricas de evaluación múltiple, como es el caso de pruebas de homogeneidad, se encontró que en general existían diferencias entre grupos en al menos en dos variables importantes - Respecto a la variable de estudios previos en Economía, donde no se determinó diferencias entre aquellos que consiguieron el doctorado y aquellos que no obtuvieron ningún grado. Sin embargo, si existirían diferencias en cuanto a estudios previos en Ciencias ambientales y también diferencias en si tenían o no un trabajo independiente; es decir hay una mayor tendencia a obtener el grado de doctor (DERN-DS) si se cuenta con estudios previos en estudios ambientales, asimismo la obtención del grado de doctor no depende del hecho de que el estudiante tenga un trabajo independiente. 
e. Usando las Pruebas de independencia con Tablas de Contingencia, se determinó que la obtención del grado de doctor depende de tener estudios formales en Ciencias Ambientales, pero es independiente de que el estudiante tenga una base en estudios de ciencias económicas y de si tiene -o no- trabajo independiente. Asimismo, existen diferencias entre los dos grupos (los que obtuvieron grado de Doctor y los que no lo obtuvieron) solo con respecto a si fueron docentes de tiempo parcial y tiempo completo. En los demás casos, de las variables: género, estudiantes con Comité asesor mixto o no, y nacionalidad, aparentemente no habría diferencias entre los dos grupos de estudio.

f. En la prueba no paramétrica de Kruskal-Wallis, para analizar las dos variables cuantitativas: promedio ponderado acumulado y número total de créditos, así como su efecto en la graduación (o no), los resultados mostraron que los promedios ponderados fueron similares entre las categorías: estudiantes con doctorado, candidatos y aquellos que no tienen ningún grado. Por otra parte, en la variable "número total de Créditos cursados", también lo resultados fueron similares en las tres categorías.

g. En definitiva, el método propuesto del uso del modelo probabilístico Logit y pruebas no paramétricas permite determinar secuencialmente variables fundamentales para este tipo de estudio, permitió el análisis partiendo de una determinación general de variables a detalles de discusión entre las variables consideradas.

h. Finalmente, según resultados presentados en la Tabla 5, el perfil óptimo de los estudiantes del Doctorado en Economía de los Recursos Naturales de la UNALM es tal que tienen estudios previos en Ciencias Ambientales, se dedican a la docencia a tiempo parcial y generalmente tienen una relación laboral de dependencia. Otras variables tales como Estudios previos en Economía, Género, Tipo de comité de tesis, Promedio acumulado de calificación y Total de créditos acumulados no hacen diferencia entre graduados y no-graduados; por lo tanto, no son variables determinantes del perfil óptimo de un ingresante con más alta probabilidad de éxito (graduación).

Tabla 5. Resumen de significancia de variables (pruebas no paramétricas)

\begin{tabular}{|c|c|c|}
\hline Obtuvieron & Variables significativas & Variables no significativas \\
\hline $\begin{array}{l}\text { Grado de } \\
\text { Doctor }\end{array}$ & $\begin{array}{c}\text { Estudios previos en ciencias } \\
\text { ambientales } \\
\text { Docente de tiempo parcial }\end{array}$ & $\begin{array}{r}\text { Estudios previos } \\
\text { Género }( \\
\text { Tipo de comi }\end{array}$ \\
\hline $\begin{array}{l}\text { Ningún } \\
\text { Grado }\end{array}$ & $\begin{array}{c}\text { Trabajo independiente } \\
\text { Docente de tiempo completo }\end{array}$ & $\begin{array}{l}\text { Promedio acumulado de } \\
\text { calificación, Total de créditos } \\
\text { acumulados. }\end{array}$ \\
\hline
\end{tabular}

\section{Literatura citada}

Alarcón, J. \& Nolazco, J. 2014. Econometría con E-views y Aplicaciones en Economía de Recursos Naturales y Desarrollo Sustentable. Fondo Editorial UNALM, Lima (Perú).
Albalate, D; Fageda, X. \& J. Perdiguero. 2011. "Éxito académico, características personales y proceso de Bolonia: una aplicación econométrica". En Revista d'Innovació Docent Universitària 2011(3), 11-25. Disponible en [http://revistes.ub.edu/index.php/RIDU/ article/view/105.000001655].

Bartual, T. \& Poblet, M. 2009. "Determinantes del rendimiento académico en estudiantes universitarios de primer año de Economía". En Formación universitaria, 3(2), 172-181. Disponible en: [http://refiedu.webs.uvigo. es/Refiedu/Vol2_3/REFIEDU_2_3_6.pdf].

García, O. \& Barrón C. 2011. "Un estudio sobre la trayectoria escolar de los estudiantes de doctorado en Pedagogía". En Perfiles Educativos XXXIII, Pg. 95. Disponible en: http://www.redalyc.org/articulo. oa? id=13218531007.

Gujarati, D. \& D. Porter (2009). Econometría. Quinta Edición, Mc Graw Hill, Santa Fé de Bogotá.

Martinez, G.; Urrutia, A.; Martinez, F.; Ponce, R.; Gil, M (2003). "Perfil del estudiante de posgrado con éxito en la UNAM". En revista Tarbiya: 2003 (32),133145. Disponible en: [https://dialnet.unirioja.es/servlet/ articulo?codigo $=713550]$.

Rosas, F. (2008). "Reconocimiento de patrones de rendimiento de los postulantes en el concurso de admisión 2005-I de la Universidad Nacional Agraria La Molina usando la técnica análisis discriminante. En revista Anales cientificos 69(2), 75-85.

Toma, J. \& Rubio, J. (2008). Estadística Aplicada, $2^{a}$ Parte. Apuntes de Estudio 69 de la Universidad del Pacífico, Lima (Perú).

Soria, K. \& Zúñiga, S. (2014). “Aspectos determinantes del éxito académico de estudiantes universitarios". En Revista Formación universitaria, 7(5), 41-50.

Wooldridge, Jeffrey (2010). Introducción a la Econometría: Un Enfoque Moderno. Tercera Edición, CENGAGE Learning ED. 